\title{
In-Silico Molecular Docking in Screening of Anti-Diabetic Therapeutics from Medicinal Plants
}

\author{
Review Article
}

\section{Shankar Gharge', Pooja Kagawad', Mahesh S Palled², Meenaxi M Maste ${ }^{2}$, Shailendra S Suryawanshi ${ }^{*}$}

1. PG Scholar, 2. Professor, 3. Assistant Professor, Department of Pharmaceutical Chemistry, KLE College of Pharmacy, KLE Academy of Higher Education and Research, Belagavi.

\begin{abstract}
One of the most widely spreading diseases due to several lifestyle problems in the 21 st century is diabetes mellitus. The management of diabetes mellitus is very important and essential. Plants are natural reservoir of many medicinal value added components help to overcome many chronic disorders including diabetes mellitus. Herbal drugs are prescribed in treatment of diabetes mellitus due to their good effectiveness, fewer side effects in clinical experience and relatively low costs. Screening of antidiabetic therapeutics is very important and essential for effective management of diabetes mellitus. Many researchers have worked on extraction, isolation, characterization of extracts and bioactive fractions from medicinal plant also they have established profile and data of interaction of active components against various targets and enzymes of diabetes mellitus using In-silico molecular docking tools. Molecular docking is an important computational tool to predict the plausible interactions between the drug and protein in a non-covalent fashion. Extensive in silico docking procedures have been carried out to examine whether the compound is a good ligand with diabetic targets. In the present review article we have thoroughly screened research articles published in various scientific, indexed, national and international journals on In-silico molecular docking based screening of Anti-Diabetic potentials and therapeutics from medicinal plant and extensively presented.
\end{abstract}

Key Words: Diabetes Mellitus, Diabetic Targets, Herbal Drugs, Molecular Docking, Protein.

\section{Introduction}

One of the most widely spreading diseases due to several lifestyle problems in the 21 st century is diabetes mellitus (DM). This disease is generally classified by insulin-dependent or type $1 \mathrm{DM}$, which is mainly initiated by destruction of the insulin producing pancreatic $\beta$-cells, and nondependent insulin or type 2 $\mathrm{DM}$, and this is triggered by lifestyle-related obesity or other exogenous components involve in it. People with type 2 diabetes are not dependent on exogenous insulin and People with type 1 diabetes need to take insulin injection for survival. In the treatment of diabetes, the drug consumption is a complementary treatment besides from diet. Oral antidiabetic drugs may be useful for people who are allergic to insulin or do not use insulin injection. And the use of these drugs in the long term has lots of disadvantages, which mainly causing increasing the risk of heart attack and acute kidney toxicity. Therefore, many efforts to develop traditional medicine for the treatment of diabetes are mounting (1).

\section{* Corresponding Author:}

\section{Shailendra S Suryawanshi}

Assistant Professor, Department of Pharmaceutical Chemistry, KLE College of Pharmacy, KLE Academy of Higher Education and Research, Belagavi-560010, Karnataka. India.

Email Id: shailendrasuryawanshi@klepharm.edu
Plants are natural reservoir of many medicinal value added components helps to overcome many chronic disorders. Hence herbal medicines are considered to be an excellent remedy for diseases like cancer, diabetes, liver diseases and arthritis. The bioactive compounds of medicinal plants like Ruellia tuberosa, Grewia hirsuta, Albizza Lebbeck benth, Phyllanthus emblica, Piper longum linn etc are used as anti-diabetic, chemotherapeutic, anti-inflammatory, anti-arthritic agents where no satisfactory cure is present in modern medicines. The bioactive compounds of medicinal plants are used as chemotherapeutic, antidiabetic, anti-arthritic agents, anti-inflammatory where no satisfactory cure is present in modern medicines.

Screening of antidiabetic therapeutics is very important and essential for effective management of DM. Many researchers have worked on extraction, isolation, characterization of extracts and bioactive fractions from medicinal plant also they have established profile and data of interaction of active components against various targets and enzymes of DM using In-silico molecular docking tools.

Molecular docking is an important computational tool to predict the possible interactions between the drug and protein in a non-covalent fashion. Extensive in silico docking procedures have been carried out to examine whether the compound is a good ligand with diabetic targets such as Aldose reductase, Peroxisome proliferatoractivated receptor-gamma, Glycogen synthase kinase-3, Pyruvate dehydrogenase 
kinase isoforms 2, Glucokinase, 11ß-Hydroxysteroid dehydrogenase, Glutamine, fructose6-phosphate, amidotransferase (2). Molecular docking was performed in the form of flexible docking for the prediction ofligand efficiency, binding affinityand the inhibitory constant. All of the ligands were docked with the target active site. Active compounds will only have positioning that avoids penalties and receives favourable scores for accurate hydrophobic contacts between the protein and ligand (3).

\section{Materials and Methods \\ Collection of Data}

The relevant information and literature is reviewed, referred and collected from various databases and journal sites. Various databases like Pubmed, Scopus, Web of Science and other related plant and medicinal plant research sites were used for searching the information and articles. Published work and literatures mainly related to "In-Silico Molecular Docking in Screening of Anti-Diabetic Therapeutics from Medicinal Plants" were collected and downloaded from journal related to medicinal plants, herbal medicines and Ayurvedic sciences and other journal related to traditional systems medicine. Published research works on molecular docking based screening of anti-diabetic potentials of plant and extracts were selected from net resources and reviewed. In the present review article, we have thoroughly screened research articles published in various scientific, indexed, national and international journals on In-silico molecular docking based screening of Anti-Diabetic potentials and therapeutics from medicinal plant and extensively reviewed.

Various research articles, data, and reports published by scientist on docking based identification and study of interaction of targets and ligands from medicinal plants were presented as follows:

Anna Safitri et al., have reported antidiabetic activity by In-Silico method by using aqueous extracts of Ruellia tuberosa L by using molecular docking using phenolic compound and the interaction between betaine, didzein and hispidulin in docking with human pancreatic $\alpha$-amylase shows different binding by hydrogen and hydrophobic bond which shows good interactions and which inhibits $\alpha$-amylase protein and shows antidiabetic activity (1).

Abirami Natarajan et al., have reported by methanolic extract of Grewia hirsuta by using molecular docking studies of ligand $(4 \mathrm{Z}, 12 \mathrm{Z})$ cyclopentadeca-4,12-dienone and docking with several different target proteins shows that it has good inhibition property, which docks well with various targets related to antidiabetic activity (2).

Prabhu Srinivasan et al., have reported which shows antidiabetic activity of quercetin by methanolic extract from Phyllanthus emblica $L$ fruitbyin-silico molecular dockingstudies which shows better docking score to glucogen phosphorylase with quercetin than with gallic acid and different concentration of quercetin shows antihyperglycemic effect and potent defense mechanism in STZ (Streptozotocin) induces antidiabetic activity (3).

Sodik Numonov et al., have reported antidiabetic activity and chemical composition of Geranium collinum root extract by molecular docking analysis suggests polyphenolic compounds such as corilagin, catechin and caffeic acid inhibit PTP-1B and $\beta$-sitosterol-30- $\beta$-D-gluco-pyranoside inhibits $\alpha$ glucosidase shows antidiabetic activity (4).

Danish Ahmed et al., have reported antidiabetic activity by molecular docking studies of some flavonoids from ethanolic extract of Albizza Lebbeck benth bark that inhibit $\alpha$-glucosidase and alpha-amylase enzyme targets to reduce glucose level and molecular docking study says binding affinity and inhibition of $\alpha$ glucosidase and $\alpha$-amylase enzyme with DPPH which shows good binding interaction shows antidiabetic activity (5).

Quy Trinch et al., have reported antidiabetic activity of bioactive compounds in Euphorbia hirta L using molecular docking shows that flavonoid and terpenes families including cyanidin,3,5-O-diglucose myricitrin, perlargonium-3-5-diglucose, quercetin, rectin, $\alpha$-amyrine, $\beta$-amyrine and taraxerol have high binding affinity with a specific enzyme receptor and shows antidiabetic activity (6).

Laura Guasch et al., have reported antidiabetic activity by PPAR-y (Potential peroxisome proliferators activated receptor gamma) partial agonist of natural origin by initial set of 29,779 natural products have proticated PPAR- $\gamma$ partial agonist 12 molecules from 11 extracts known to have antidiabetic activity (7).

Juan Jose Ramirez Espinosa et al., have reported antidiabetic activity by pentacyclic acid triterpenoid's role of PTP-1B by In-Silicomethod and related triterpinic acid, anolic, moronic, morolic acid which shows extensive hydrogen bond network with carboxyl group and Vander Waals interactions stabilizes the protein-ligand complex which shows antidiabetic activity (8).

Bikash Thakuria et al., have reported bioinformatics-based investigation to screen and analyze the bioactivity of Piper longum linn, which shows antidiabetic activity and the ligands of piperine, retrofractamide A, piper-longumine indicated great docking with piperine shows $-8.69 \mathrm{kcal} / \mathrm{mol}$ restricting vitality of $0.429 \mu \mathrm{m}$ hindrance which has greatest potential to be decent inhibitor against focused receptors which shows antidiabetic activity (9).

Aristeidis Pritsas et al., have reported volarisation of stachysetin from cultivated Stachys ira Griseb which shows antidiabetic agent, in silico screening against 17 proteins implicated in diabetes as also ligand-based similarity metrics against established antidiabetic activity drug and stachysetin shows binding profile to major drug carrier plasma protein serum albumin was explored along with its properties shows antidiabetic activity (10).

Zoy I Noor et al., have reported in-silico molecular docking stimulations with $\alpha$-amylase and $\alpha$ lipase enzyme of polar and non-polar extracts of leaves and flowers Ocimum basilicum andIn-Silicostudy 
linalool and estragole to have considerable porcine pancreatic $\alpha$-amylase (PPA) and pocrine pancreatic lipase (PPL) binding potential and which further investigated through molecular dynamics and binding free energy calculations which shows best antidiabetic activity (11).

Abhijit Pathak et al., have reported in silico molecular docking analysis of isolated compounds of Ocimum sanctum against antidiabetic activity and a wide range of docking score found during molecular docking by maestro v 10.1 (Schrodinger) and among them carvacrol had lowest docking score against $\alpha$ amylase enzymes and glucokinase which is $-50521 \mathrm{kj} /$ $\mathrm{mol}$ and $-7.322 \mathrm{kj} / \mathrm{mol}$ and this is concluded less docking score compound will be more potent and shows antidiabetic activity (12).

T. Hoang Nguyen Vo et al., have reported insilico molecular docking analysis from Euphorbia thymifolia it has 7 compounds were chosen due to high absolute value of binding energy to all four receptors (>8 $\mathrm{kcal} / \mathrm{mol}$ ) known as $\beta$-amyrine,toraxerol,1-Ogalloyl- $\beta$-D-glucose ,corilagin,cosmossin,quercetin-3galactoside and quercetin ,tannins, polyphenols and flavonoid family had high binding affinity to all receptors beside that, the binding affinity of two of terpenoid compounds has good prospect for treatment of type-2 diabetes mellitus (13).

Jasmine $\mathrm{R}$ et al., have reported antidiabetic activity in Streptozotocin (STZ) diabetic rats by insilico method of molecular screening method which shows antidiabetic activity against target proteins like PPAR- $\gamma$ which plays a role in protecting $\beta$-cells from damage was undertaken and docking analysis in active site of $2 \mathrm{PRG}$ was performed by Schrodinger program and it shows good binding interactions of ligands with targets at low energy level from terpenoid from Elephantopus scaber $L$, it increases insulin secretion from regenerated pancreatic $\beta$ cells which is potent antidiabetic activity (14).

G. Mahendran et al., have reported in-silico antidiabetic activity isolated from Swertia corymbose which having 1,2,8-trihydroxy-6-methoxy xanthone (ligand 1) and 1,2-dihydroxy-6-methoxy xanthone-8-O$\beta$-D-xylo pyranosyl (ligand 2) isolated from Swertia corymbosewhich shows antidiabetic activity by molecular docking studies by STZ(Streptozotocin) induced diabetic rats and ligand 1 and 2 and glibendamide with various diabetics docked with glucokinase , fructose-1,6-biphosphatase 1 with sulfonyl urea receptor shows good binding activity towards antidiabetic activity (15).

Md. Nazmul Prottoy et al., have reported by molecular docking pharmacological property analysis of an antidiabetic activity of some medicinal plants of Bangladesh against type 2 diabetes by In-Silico computational approach and some of them are Aegeline from Aegle maemelos, gallic acid from Terminalia bellirica, quercetin from Psidium guajava and mangiferin from Mangifera indica have antidiabetic activity among them quercetin have greatest source of antidiabetic activity (16).
Ranjit $\mathrm{D}$ et al., have reported In-Silico antidiabetic activity of bioactive compound in Ipomoea mauritiana jack which shows molecular interactions by using Argus lab docking software4.0.1 among this taraxerol shown maximum inhibition for $3 \mathrm{G} 9 \mathrm{E}$ protein both shows antidiabetic activity (17).

Pradeep Paudel et al., have reported antidiabetic activity by In-Silico molecular docking study of 2.3.6-tribromo-4.5-dihydroxybenzyl derivatives from marine algae Symphyodadia latiuscula through PTP1B (tyrosine phosphate 1 B) down regulation and $\alpha$-glucosidase inhibition which shows antidiabetic activity (18).

Yue Chen et al., have reported by In-Silico molecular docking studies which shows antidiabetic activity from Ellagitannins isolated compound from unripe fruit Kubus chingii $h u$ which shows molecular docking results revealed that Ching tannin A interacted with enzymes mainly by $\mathrm{H}$-bond and was bound in allosteric site which shows good interaction and act as antidiabetic activity (19).

Jirawat Riyaphan et al., have reported hypoglycemic efficacy of In-Silico molecular docking selected natural compounds against $\alpha$-glucosidase and $\alpha$-amylase via molecular docking and enzymatic measurement on $\mathrm{CaCo}-2$ cell and act as antidiabetic agent (20).

Rangachari Balamurugan et al., have reported $\mathrm{\gamma}$-sitosterol isolated from Lippia nodiflora was taken as ligand for molecular docking and the targets like glucokinase, fructose-1,6-biphosphatase 1, by human multidrug resistance protein 1 and Cytochrome P 450 by autodock tool v 4.2 and APT V 1.5.4 program and $\mathrm{y}$ sitosterol which deals well with various targets related to diabetes mellitus (21).

Priyanka Sharma et al., have reported in-silico screening of potential antidiabetic activity from Phyllanthus emblicaagainst the type-2 diabetes and docking score and pharmacophore studies found that ellagic acid, estradiol, sesamine, kaempferol, zeatin, quercetin and leucodelphinidin are potential antidiabetic activity (22).

Hyun Ah Jung et al., have reported molecular docking studies of an antidiabetic complication inhibitor such as fecosterol from edible brown algae Eisenia bicyclis and Ecklonia stilonifera which shows evaluating ability of this compound to inhibit rat lense aldose reductase (RLAR), human recombinant aldose reductase (HRAR), protein tyrosine phosphatase (PTP1B) and $\alpha$-glucosidase which shows binding energy $(-8.2 \mathrm{kcal} / \mathrm{mol})$ for RLAR and $(-8.5 \mathrm{kcal} / \mathrm{mol})$ for HRAR shows antidiabetic activity (23).

Andreia S.P. Pereira et al., have reported antidiabetic activity of some common herbs and species by providing them by some In-Silicovirtual screening method by in-silico method by using antidiabetic drug targets such as achillin B from yarrow, asparasaponin I from fenugreek, bisdemothoxy curcumin from turmeric , carlinoside from lemon grass with major protein targets like dipeptidyl-peptidase-4(DPP4), intestinal maltase-glucoamylase liver receptor alpha ,protein tyrosine phosphatase non-receptor type 
interaction which indicates antidiabetic potential of common herbs and species (24).

Mingzhu Jiang et al., have reported In-Silico molecular docking study with $\alpha$-glucosidase inhibitory peptides from Soybean protein hydrolysate which significantly reduces levels of fasting blood glucose in mice and this confirms that $\alpha$-glucosidase inhibitory peptide may have hypoglycemic efficacy (25).

Nahid Ghaedi et al., have reported antidiabetic activity of alcoholic extract of leaf and stem of Levisticum officinalae of an implication for $\alpha$-amylase inhibitory activity of extract ingredient by molecular docking method that which shows antihyperglycemic effect of it among them lueteolin, quercetin, rosmarinic, caffeic and hexanoic acids have greatest $\alpha$-amylase inhibition activity (26).

Sarfaraz Ahmed et al., have reported InSilicomolecular docking studies on miquelianin isolated from aerial part of Euphobia schimperi c exhibited significant results for antidiabetic potential and miquelianin which significant $\alpha$-amylase and $\alpha$ glucosidase inhibitory activity which shows antidiabetic activity (27).

Vineet Mehta et al., have reported antidiabetic activity of hydroalcoholic extract from Ocimumcanctum which says greatly inhibited $\alpha$-glucosidase enzyme but failed to inhibit $\alpha$-amylase activity and docking studies predicted that rosmarinic acid, stigmasterol, linalool, aesculin may be responsible for antidiabetic activity possessed by plant through their interaction with insulin receptor (28).

P. Rajkumar et al., have reported antidiabetic activity compounds from the flowers of Cassiaauriculata by structure based molecular docking studies, which says that docking results showed best glide energy, docking score H-bonding interactions compared with molecular targets and has potential to prevent or treat type-2 diabetesmellitus (29).

Farhat Saghir et al., have reported In-Silico molecular docking screening studies from hexane extract of Pongamia pinnata flower which shows antidiabetic activity and molecular docking studies which indicates high binding energy scored with antidiabetic targets as compared to standard drug acarbose and results shows isolated compound 1(4methoxy-7-phenyl-5H-furo [3,2,9][1] benzopyran-5one) has antidiabetic activity (30).

Jae Sue Choi et al., have reported antidiabetic activity of protein tyrosine phosphatase $1 \mathrm{~B}$ inhibitor activity of alkaloids from Rhizoma Coptidis by molecular docking studies which conclude that alkaloids of Rhizoma coptidis (berberine, magnoglorine, coptisine, epiberberine) exhibited remarkable inhibitory activities against PTP1B which has good binding affinity and docking score against PTP1B which shows antidiabetic activity (31).

Sudhanshu Kumar Bharti et al., have reported antidiabetic activity of fruto and isomal to oligosaccharides by In-Silicostudies by docking were performed by GLIDE program for each F O S ( Frutooligos a c harides) and IMO'S(Isomaltooligosaccharides) for PPAR-y activation and DPP-IV inhibition which shows antidiabetic activity and the FOS was produced from Aspergillus oryzae and IMO'S and standards for 1kestose,1-nystose,1-frutofuranosyl nystose and panose were procured (32).

Vikas Kumar et al., have reported which shows hypoglycemic effect of wedeloketone isolated from Wedelia calendulacea by In-Silicomolecular docking against dipeptidyl peptidase-4(DPP4), glucose transporter-1(GLUT1) and peroxisome proliferator activated receptor- $\gamma($ PARA- $y)$ which shows docking score near $-6.17,-9.43$ and -7.66 respectively and wedelolactone treat type-2 diabetesmellitus (33).

Abdul Sadiq et al., have reported hyperglycemic activity from Eryngium caeruleum $M$. Bieb by $\alpha$-glucosidase by molecular docking studies shows explore possible role of all identified bioactive compound in chloroform fraction of Eryngium caeruleum M. Bieb into active sites of homology model of $\alpha$-glucosidase which shows antidiabetic activity (34).

Chien-Hung Jhong et al., have reported byInSilicomolecular dockingstudies of $\alpha$-glucosidase and $\alpha$ amylase inhibitors from natural compounds by molecular docking in-silico and correlation analysis indicated that curcumin and actinodaphinine had high activity $\alpha$-glucosidase as well as curcumin and berberine for $\alpha$-amylase inhibitors when compared with acarbose which shows antidiabetic activity (35).

Jayasree Ganugapati et al., have reported InSilico molecular screening studies of banana flower flavonoids as insulin receptor tyrosine kinase activators as cure for diabetes mellitus by Autodock Vina, Autodock 4.0.to phosphorylated tyrosines docked with hesperitin triacetate, naringenin, naringenin pelargonidin and naringenin flavanone are potent activators of IR tyrosine kinase which shows antidiabetic activity (36).

Noor Rahman et al., have reported In-Silico molecular docking studies of isolated alakaloids for $\alpha$ glucosidase inhibition compared with standard acarbose and miglitol were docked to $\alpha$-glucosidase by using MOE-Dock applied in MOE software to predict the binding modes of these drug-like compounds which shows antidiabetic activity (37).

Muhammad Nadeem et al., have reported antidiabetic byIn-Silicomolecular dockingstudies of potential of leaf extracts and an insight into molecular docking by ethanolic extract of Calotropis procera and which inhibit $\alpha$-glucosidase and $\alpha$-amylase synergistically to prevent hyperglycemic activity (38).

Yang Yang et al., have reported antidiabetic activity byIn-Silicomolecular dockingstudies of potential dipeptidyl peptidase (DPP)-IV inhibitor among Moringa oleifera by phytochemical virtual screening and molecular docking analysis was used to stimulated the interaction mode of candidate compounds with DPP-IV receptors which shows antidiabetic activity (39).

Poonam Kalhotra et al., have reported antidiabetic activity by molecular docking of natural product library reveal chrysin as a novel Dipeptidyl peptidase-IV (DPP-IV) inhibitor by in-silico method 
and in-vitro assay revealed that chrysin inhibits DPP-IV enzyme in a concentration dependent manner shows antidiabetic activity (40).

Olusola Abiola ladokun et al., have reported potent antidiabetic activity of methanolic extract of Hunteria umbellata by molecular docking studies and its compound 2.2-Benzylidenebis (3-methyl benzofuran) have significant antidiabetic activity against PPAR-y and molecular binding interaction shows potent antidiabetic activity (41).

Ajmer Singh Grewal et al., have reported InSilico molecular docking studies of phenolic compounds from Syzygium cumini by multiple targets of type-2 diabetes and act as antidiabetic agent by insilico docking study includes $\alpha$-glucosidase, dipeptidyl peptidase, glycogen synthtase kinase, glucagon receptor catechin and myricetin, quercetin was found to inhibit DPP-IV and develop safe and potent natural type-2 antidiabetic activity (42).

Usman Ghani et al., have reported antidiabetic activity by In-Silico molecular docking studies by natural flavonoid $\alpha$-glucosidase inhibitors from Retama raetam by molecular docking interaction with enzyme active site and Retamasin D, G, H and Erysubin A and B non-competitively inhibited the enzyme whereas retamasin $\mathrm{C}$ and $\mathrm{F}$ exhibited competitive inhibition which inhibits $\alpha$-glucosidase enzyme active site shows antidiabetic activity (43).

Arumugam Sudha et al., have reported In-Silico molecular docking studies of 1,2 disubstituted idopyranose from Vitex negundo by its antidiabetic activity of type-II diabetes .it manly targets proteins active site (dipeptidyl peptidase-IV and glycogen synthtase kinase-III) which results in inhibition of enzyme active site, the binding energy of ligand protein interactions also confirmed that its inhibitory activity (44).

Sudipta Ghosh et al., have reported In-Silico molecular docking and inhibition studies of $\alpha$-amylase activity by Labdane diterpenes from Alpinia nigra seeds two labdane diterpenes (I and II) for $\alpha$-amylase inhibitor activity by comparing with standard acarbose among I and II, the diterpene shows highest MolDock and rerank score to show antidiabetic activity (45).

Pawan Kaushik et al., have reported antidiabetic activity by In-Silico molecular docking studies which shows pharmacophore modeling and molecular docking studies on Pinus roxburghii as target for diabetes mellitus and 17 constituents from Pinus roxburghii were docked on different receptors out of which secoisoresinol, pinoresinol and cedeodarin showed highest affinity for the aldose reductase and targets are protein tyrosine phosphate-1$\beta(\mathrm{PTP}-1 \beta)$,dipeptidyl peptidase-IV (DPP-IV), aldose reductase (AR) and insulin receptor with help of docking software Molegro virtual docker(MVD) results of docking score which shows antidiabetic activity(46).

Narasimhamurthy Konappa et al., have reported antidiabetic activity by In-Silico molecular docking studies of Amomum nilgiricum by molecular docking interactions of bioactive serverogenin acetate with target proteins and docking studies were carried out for
Phyto ligands using iGEMDock program to elucidate the binding affinities to target proteins targets are $\alpha$ amylase and $\alpha$-glucosidase and ligand is serverogenin acetate shows antidiabetic activity (47).

Nausheen Nazir et al., have reported antidiabetic activity byIn-Silico molecular docking studies of Elaeagnus umbellate in Streptozotocin (STZ) induced diabetic rat. Chloroform, ethyalacetate extract of potent controlling hyperglycemia in STZ induced type-II diabetes in rats. Molecular docking approach indicated the favorable inhibitory interaction between docked compounds and active site of $\alpha$-glucosidase and $\alpha$-amylase and cocked compounds occupy binding site as occupied standard acarbose (48).

Da Hye Kim et al., have reported In-Silico molecular docking studies by potential of icarrin metabolites from Epimedium koreanumNakai as antidiabetic activity, icariin, its deglycosylated icaritin and glycosylated flavonoids (icaeriside II, epimedinA, epimedinB, epimedin C) were evaluated their ability to inhibit protein tyrosine phosphatase $1 \mathrm{~B}(\mathrm{PTP} 1 \mathrm{~B})$ and $\alpha-$ glucosidase. Furthermore, enzyme kinetics analysis and molecular docking shows antidiabetic activity (49).

Chunsheng Zhy et al., have reported antidiabetic activity In-Silico molecular docking studies towards $\alpha$-glucosidase inhibitor from Clerodendranthus spicatus based on HSCCC coupled by molecular docking method. Among five compounds like 2caffeoyl-L-tartaric acid, N-(E)-Caffeoyl dopamine, rosmarincacid, methyl rosmarinate, $6,7,8,3^{\prime}, 4$ pentamethoxy flavone and molecular docking indicated that the affinity energy of identified compounds among them rosmarinc caid which shows antidiabetic activity (50).

Dhiraj Kumar Choudhary et al., have reported In-Silico molecular docking interaction of methanol, acetone extract which gives porcine from Vicia faba crude seed extract and evaluate antidiabetic activity with $\alpha$-amylase by hydrogen bonding and hydrophobic interaction which shows antidiabetic activity (51).

Rina Herowati et al., have reported antidiabetic activity by molecular docking studies of chemical composition of Tinospora cordifolia on glycogen phosphorylase results indicated that Autodock-vina's algorithms were valid and the docking result revealed that magnoflorin, cardifoliside A and syrimgin exhibited good binding interaction with active site glycogen phosphorylase shows better anti-diabetic activity (52).

Yan Wang et al., have reported molecular docking screening for identifying hyperoside as an inhibitor of fatty acid binding protein 4 from a natural product and the report as flavanols to be ideal scoffold for FABP4 inhibitor development among popular flavanol (53).

Sudhanshu Kumar bharti et al., have reported antihyperglycemic activity by in-silico molecular docking studies with DPP-IV inhibition of alkaloids from seed extract of Castanospermum austrate by molecular docking studies and berberine which shows competitive inhibition towards DPP-IV by molecular docking studies to normalizes hyperglycemia in type-II 
diabetes mellitus rats with strong DPP-IV inhibitory potential activity (54).

Samuel Odeyemi et al., have reported antidiabetic activity by in-silico molecular docking studies by affecting glucose uptake in HepG2 cells following the exposure to methanolic extract of Lauridia tetragona and the $\alpha$-glucosidase, $\alpha$-amylase, dipeptidyl peptidase-IV (DPP-IV), lipase inhibitory activities and glucose uptake in HepG2were investigated which shows good hypoglycemic activity that may be linked to inhibition of crucial enzyme associated with diabetes (55).

Tae Kyung Hyun et al., have reported antidiabetic activity by molecular docking studies for discovery of plant derived $\alpha$-glucosidase inhibitors which mainly treat type-II diabetes mellitus and $\alpha$ glucosidase docked with rectine, quercetin and myricetin these can be good patent of $\alpha$-glucosidase inhibitors act as antidiabetic activity (56).

Channabasava et al., have reported antidiabetic activity by using in-silico molecular dockingstudies of methanolic extracts of Loranthus micranthus by GEM Dock method and common phytochemical in all extract is octadecenoic acid used separated and used as antidiabetic activity (57).

Pukar Khanal et al., have reported antidiabetic activity by using In-Silico molecular docking studies of Tinospora cordifolia interaction between the compounds, proteins and pathway was interpreted based on edge count. The docking study was performed using Autodock 4.0. and the binding affinity and inhibitory constant of tembetarine with $\beta$-1-adrenergic receptor was found to be $6.25 \mathrm{kcal} / \mathrm{mol}$ which shows antidiabetic activity (58).

Kiran Kumar Angadi et al., have reported InSilico molecular docking studies of guggultetrol from Nymphaea pubescens with target glucokinase related to type-II diabetes which shows good bond interactions by In-Silico method as antidiabetic activity (59).

Muhammad Raza Shah et al., have reported antidiabetic activity by using In-Silico molecular docking studies in which shown by protein tyrosine phosphatase 1B inhibitors isolated from Artemisia roxburghiana and antidiabetic activity of Artemisia roxburghiana could be activated due to PTP1B inhibition by its triterpene constituents like botulin, betulinic acid and taraxeryl acetate against tyrosine phosphatase 1B protein receptor which shows antidiabetic activity (60).

Nur Athirah Zabidi et al., have reported antidiabetic activity by using In-Silico molecular docking of inhibitory evaluation of Curculigo latifolia on $\alpha$-glucosidase and results phlorin binds strongly with enzyme receptor achieving the lowest binding energy value effective in lowering hyperglycemia (61).

Sathianpong phoopa et al., have reported antidiabetic activity by using In-Silico molecular docking by chemical constituents of Litsea elliptica and their $\alpha$-glucosidase inhibition with in-silico method by molecular docking studies and quercetin diglycoside isolated and docked with $\alpha$-glucosidase enzyme receptor shows antidiabetic activity (62).

\section{Discussion}

Diabetes Mellitus is widely spreading diseases due to several lifestyle problems in the 21 st century. Treatment and prevention of diabetes is very much essential. Plants are natural reservoir of many medicinal value added components helps to overcome many chronic disorders. Hence herbal medicines are considered to be an excellent remedy for treatment of diabetes mellitus. Molecular docking is an important computational tool to predict the possible interactions between the drug and protein in a non-covalent fashion. Extensive in silico docking procedures have been carried out to examine whether the compound is a good ligand with diabetic targets. Screening of antidiabetic therapeutics is very important and essential for effective management of DM. Many researchers have worked on extraction, isolation, characterization of extracts and bioactive fractions from medicinal plant also they have established profile and data of interaction of active components against various targets and enzymes of DM using In-silico molecular docking tools.

The reviewed articles have extensively concentrated on use of different tools and methods of molecular docking technique. They have extracted, isolated and screened various plant constituents against various targets of DM. Screening included the utilization of different plants from various families and they have isolated constituents which are belongs to the different chemical classes like, alkaloids, glycosides, flavonoids, tannins and diterpene class. Hence the reviewed methods are valuable in identification of important therapeutics from medicinal plants for effective management of DM.

\section{Conclusion}

The present review concludes that the management of diabetes mellitus is very important and essential and medicinal plants are natural reservoir of many therapeutic value added components helps to overcome many chronic disorders including diabetes mellitus. In-Silico molecular docking studies and related computational tools are very important and essential in the screening of Anti-Diabetic therapeutics from medicinal plants. Extensive In-Silico docking procedures have been carried out to examine whether the compound is a good ligand with diabetic targets which helps to identify new and important therapeutics for management of diabetes mellitus.

\section{Conflict of Interest}

$$
\text { Nil }
$$

\section{Abbreviations}

1. STZ -Streptozotocin

2. PPAR-y -Potential peroxisome proliferators activated receptor gamma

3. PTP1B -tyrosine phosphatase 1B

4. DPP-IV-dipeptidyl peptidase-IV

5. MVD-Molegro virtual docker

6. PPL-pocrine pancreatic lipase

7. RLAR-rat lense aldose reductase

8. HRAR-human recombinant aldose reductase 
9. PTP1B- protein tyrosine phosphatase

10.PPL-pocrine pancreatic lipase

11.DM: Diabetes Mellitus

\section{References}

1. Safitri A, Fatchiyah F, Sari DR, Roosdiana A. Phytochemical screening, in vitro anti-oxidant activity, and in silico anti-diabetic activity of aqueous extracts of Ruellia tuberosa L. Journal of Applied Pharmaceutical Science. 2020 Mar;10(3):101-8.

2. Natarajan A, Sugumar S, Bitragunta S, Balasubramanyan N. Molecular docking studies of (4 Z, 12 Z)-cyclopentadeca-4, 12-dienone from Grewia hirsuta with some targets related to type 2 diabetes. BMC complementary and alternative medicine. 2015 Dec;15(1):1-8.

3. Srinivasan $\mathrm{P}$, Vijayakumar S, Kothandaraman S, Palani M. Anti-diabetic activity of quercetin extracted from Phyllanthus emblica L. fruit: In silico and in vivo approaches. Journal of pharmaceutical analysis. 2018 Apr 1;8(2):109-18.

4. Numonov S, Edirs S, Bobakulov K, Qureshi MN, Bozorov K, Sharopov F, Setzer WN, Zhao H, Habasi M, Sharofova M, Aisa HA. Evaluation of the antidiabetic activity and chemical composition of Geranium collinum root extractsComputational and experimental investigations. Molecules. 2017 Jun;22(6):983.

5. Ahmed D, Kumar V, Sharma M, Verma A. Target guided isolation, in-vitro antidiabetic, antioxidant activity and molecular docking studies of some flavonoids from Albizzia Lebbeck Benth. bark. BMC complementary and alternative medicine. 2014 Dec;14(1):1-3.

6. Trinh Q, Le L. An investigation of antidiabetic activities of bioactive compounds in Euphorbia hirta Linn using molecular docking and pharmacophore. Medicinal Chemistry Research. 2014 Apr;23(4):2033-45.

7. Guasch L, Sala E, Mulero M, Valls C, Salvadó MJ, Pujadas G, Garcia-Vallvé S. Identification of PPARgamma partial agonists of natural origin (II): in silico prediction in natural extracts with known antidiabetic activity. PLoS One. 2013 Feb 6;8(2):e55889.

8. Ramírez-Espinosa JJ, Rios MY, López-Martínez S, López-Vallejo F, Medina-Franco JL, Paoli P, Camici G, Navarrete-Vázquez G, Ortiz-Andrade R, Estrada-Soto $\mathrm{S}$. Antidiabetic activity of some pentacyclic acid triterpenoids, role of PTP-1B: In vitro, in silico, and in vivo approaches. European journal of medicinal chemistry. 2011 Jun $1 ; 46(6): 2243-51$.

9. Thakuria B, Laskar S, Adhikari S. A bioinformaticsbased investigation to screen and analyze the bioactivity of Piper longum Linn. compounds as a ground-breaking hostile to antidiabetic activity. Pharmacognosy Magazine. 2020 Mar 1;16(68):199.

10. Pritsas A, Tomou EM, Tsitsigianni E, Papaemmanouil CD, Diamantis DA, Chatzopoulou
P, Tzakos AG, Skaltsa H. Valorisation of stachysetin from cultivated Stachys ivaGriseb. as anti-diabetic agent: A multi-spectroscopic and molecular docking approach. Journal of Biomolecular Structure and Dynamics. 2020 Jul 29:1-5.

11. Noor ZI, Ahmed D, Rehman HM, Qamar MT, Froeyen M, Ahmad S, Mirza MU. In vitro antidiabetic, anti-obesity and antioxidant analysis of Ocimumbasilicum aerial biomass and in silico molecular docking simulations with alpha-amylase and lipase enzymes. Biology. 2019 Dec;8(4):92.

12. Pathak A, Nahid S, Manik AS, Rahman MS, Rumpa FT, Majumder M, Paul A. Insilico molecular docking analysis of isolated compounds of Ocimum sanctum against two related targets to diabetes. The Pharma Innovation Journal. 2017;6(9):148-51.

13. Vo TH, Tran N, Nguyen D, Le L. An in silico study on antidiabetic activity of bioactive compounds in Euphorbia thymifolia Linn. SpringerPlus. 2016 Dec;5(1):1-3.

14. Jasmine R, Kumar GA, Rajaram R. Probing the mechanism of the anti-diabetic potential of a terpenoid from Elephantopusscaber L., an Indian ethnomedicinal plant in STZ diabetic rats-In vivo and in silico analysis.

15. Mahendran G, Manoj M, Murugesh E, Kumar RS, Shanmughavel P, Prasad KR, Bai VN. In vivo antidiabetic, antioxidant and molecular docking studies of 1,2,8-trihydroxy-6-methoxy xanthone and 1,2dihydroxy-6-methoxyxanthone-8-O- $\beta$-D xylopyranosyl isolated from Swertia corymbosa. Phytomedicine. 2014 Sep 25;21(11):1237-48.

16. Prottoy NI, Sarkar B, Ullah A, Hossain S, Boby AS, Araf Y. Molecular docking and pharmacological property analysis of antidiabetic agents from medicinal plants of Bangladesh against type II diabetes: a computational approach. PharmaTutor. 2019 Sep 1;7(9):6-15.

17. Ranjith D, Viswanath S. In silico antidiabetic activity of bioactive compounds in Ipomoea mauritiana Jacq. The Pharma Innovation Journal. 2019;8(10):05-11.

18. Paudel P, Seong SH, Park HJ, Jung HA, Choi JS. Anti-diabetic activity of 2, 3, 6-tribromo-4, 5dihydroxybenzyl derivatives from Symphyocladialatiuscula through PTP1B downregulation and $\alpha$-glucosidase inhibition. Marine drugs. 2019 Mar;17(3):166.

19. Chen Y, Chen Z, Guo Q, Gao X, Ma Q, Xue Z, Ferri $N$, Zhang $M$, Chen $H$. Identification of ellagitannins in the unripe fruit of Rubus Chingii $\mathrm{Hu}$ and evaluation of its potential antidiabetic activity. Journal of agricultural and food chemistry. 2019 Jun 3;67(25):7025-39.

20. Riyaphan J, Jhong CH, Lin SR, Chang CH, Tsai MJ, Lee DN, Sung PJ, Leong MK, Weng CF. Hypoglycemic efficacy of docking selected natural compounds against $\alpha$-Glucosidase and $\alpha$-Amylase. Molecules. 2018 Sep;23(9):2260.

21. Balamurugan R, Stalin A, Ignacimuthu $\mathrm{S}$. Molecular docking of $\gamma$-sitosterol with some targets 
related to diabetes. European journal of medicinal chemistry. 2012 Jan 1;47:38-43

22. Sharma P, Joshi T, Joshi T, Chandra S, Tamta S. In silico screening of potential antidiabetic phytochemicals from Phyllanthus emblica against therapeutic targets of type 2 diabetes. Journal of ethnopharmacology. 2020 Feb 10;248:112268.

23. Jung HA, Islam MN, Lee CM, Oh SH, Lee $\mathrm{S}$, Jung JH, Choi JS. Kinetics and molecular docking studies of an anti-diabetic complication inhibitor fucosterol from edible brown algae Eisenia bicyclis and Ecklonia stolonifera. Chemico-biological interactions. 2013 Oct 25;206(1):55-62.

24. Pereira AS, Banegas-Luna AJ, Peña-García J, Pérez-Sánchez H, Apostolides Z. Evaluation of the anti-diabetic activity of some common herbs and spices: providing new insights with inverse virtual screening. Molecules. 2019 Jan;24(22):4030.

25. Jiang M, Yan H, He R, Ma Y. Purification and a molecular docking study of $\alpha$-glucosidaseinhibitory peptides from a soybean protein hydrolysate with ultrasonic pretreatment. European Food Research and Technology. 2018 Nov;244(11):1995-2005.

26. Ghaedi N, Pouraboli I, Askari N. Antidiabetic properties of hydroalcoholic leaf and stem extract of Levisticum officinale: an implication for $\alpha$ amylase inhibitory activity of extract ingredients through molecular docking. Iranian Journal of Pharmaceutical Research: IJPR. 2020;19(1):231.

27. Ahmed S, Al-Rehaily AJ, Alam P, Alqahtani AS, Hidayatullah S, Rehman MT, Mothana RA, Abbas SS, Khan MU, Khalid JM, Siddiqui NA. Antidiabetic, antioxidant, molecular docking and HPTLC analysis of miquelianin isolated from Euphorbia schimperi C. Presl. Saudi Pharmaceutical Journal. 2019 Jul 1;27(5):655-63.

28. Mehta V, Sharma A, Kailkhura PA, Malairaman UD. Antioxidant, anti-inflammatory, and antidiabetic activity of hydroalcoholic extract of Ocimum sanctum: an in-vitro and in-silico study.

29. Rajkumar P, Selvaraj S, Suganya R, Velmurugan D, Kumaresan S. GC-MS characterization of the antidiabetic compounds from the flowers of cassia auriculata (AVARAM): A structure based molecular docking studies. Int. J. Innov. Res. Sci. Eng. Technol. 2016;1:85-93.

30. Saghir F, Hussain K, Tahir MN, Raza SA, Shehzadi N, Iftikhar S, Shaukat A, Naheed S, Siddique S. Antidiabetic Screening, Activity-guided Isolation and Molecular Docking Studies of Flower Extracts of Pongamia pinnata (L.) Pierre. Journal of Medicinal plants and By-product. 2020 Dec 13.

31. Choi JS, Ali MY, Jung HA, Oh SH, Choi RJ, Kim EJ. Protein tyrosine phosphatase 1B inhibitory activity of alkaloids from RhizomaCoptidis and their molecular docking studies. Journal of ethnopharmacology. 2015 Aug 2;171:28-36.

32. Bharti SK, Krishnan S, Kumar A, Gupta AK, Ghosh AK, Kumar A. Mechanism-based antidiabetic activity of Fructo-and isomalto-oligosaccharides: Validation by in vivo, in silico and in vitro interaction potential. Process Biochemistry. 2015 Feb 1;50(2):317-27.

33. Kumar V, Sharma K, Ahmed B, Al-Abbasi FA, Anwar F, Verma A. Deconvoluting the dual hypoglycemic effect of wedelolactone isolated from Wedeliacalendulacea: investigation via experimental validation and molecular docking. RSC advances. 2018;8(32):18180-96.

34. Sadiq A, Rashid U, Ahmad S, Zahoor M, AlAjmi MF, Ullah R, Noman OM, Ullah F, Ayaz M, Khan I, Islam ZU. Treating Hyperglycemia From Eryngium caeruleum M. Bieb: In-vitro $\alpha$-Glucosidase, Antioxidant, in-vivo Antidiabetic and Molecular Docking-Based Approaches. Frontiers in chemistry. 2020 Nov 26;8:1064

35. Jhong CH, Riyaphan J, Lin SH, Chia YC, Weng CF. Screening alpha-glucosidase and alpha-amylase inhibitors from natural compounds by molecular docking in silico. Biofactors. $2015 \mathrm{Jul}$ 8;41(4):242-51.

36. Ganugapati J, Baldwa A, Lalani S. Molecular docking studies of banana flower flavonoids as insulin receptor tyrosine kinase activators as a cure for diabetes mellitus. Bioinformation. 2012;8(5):216.

37. Rahman N, Muhammad I, Khan H, Aschner M, Filosa R, Daglia M. Molecular docking of isolated alkaloids for possible $\alpha$-glucosidase inhibition. Biomolecules. 2019 Oct;9(10):544.

38. Nadeem M, Mumtaz MW, Danish M, Rashid U, Mukhtar H, Anwar F, Raza SA. Calotropis procera: UHPLC-QTOF-MS/MS based profiling of bioactives, antioxidant and anti-diabetic potential of leaf extracts and an insight into molecular docking. Journal of Food Measurement and Characterization. 2019 Dec;13(4):3206-20.

39. Yang Y, Shi CY, Xie J, Dai JH, He SL, Tian Y. Identification of potential dipeptidyl peptidase (DPP)-IV inhibitors among Moringa oleifera phytochemicals by virtual screening, molecular docking analysis, ADME/T-based prediction, and in vitro analyses. Molecules. 2020 Jan;25(1):189.

40. Kalhotra P, Chittepu VC, Osorio-Revilla G, Gallardo-Velázquez T. Structure-activity relationship and molecular docking of natural product library reveal Chrysin as a novel dipeptidyl peptidase-4 (DPP-4) inhibitor: An integrated in silico and in vitro study. Molecules. 2018 Jun;23(6):1368.

41. Ladokun OA, Abiola A, Okikiola D, Ayodeji F. GCMS and molecular docking studies of Hunteriaumbellata methanolic extract as a potent anti-diabetic. Informatics in Medicine Unlocked. 2018 Jan 1;13:1-8.

42. Grewal AS, Sharma N, Singh S, Arora S. Molecular docking studies of phenolic compounds from Syzygiumcumini with multiple targets of type 2 diabetes.

43. Ghani U, Nur-e-Alam M, Yousaf M, Ul-Haq Z, Noman OM, Al-Rehaily AJ. Natural flavonoid $\alpha$ glucosidase inhibitors from Retama raetam: 
Enzyme inhibition and molecular docking reveal important interactions with the enzyme active site. Bioorganic chemistry. 2019 Jun 1;87:736-42.

44. Sudha A, Manikandan R, Arulvasu C, Srinivasan P. Molecular Docking Studies Of 1, 2 Disubstituted Idopyranose From Vitex Negundo With AntiDiabetic Activity Of Type 2 Diabetes. Intern. J. Pharma and Bio Sci.-2011.-2.(1).

45. Ghosh S, Rangan L. Molecular docking and inhibition studies of $\alpha$-amylase activity by labdane diterpenes from Alpinia nigra seeds. Medicinal Chemistry Research. 2014 Nov;23(11):4836-52.

46. Kaushik P, Lal Khokra S, Rana AC, Kaushik D. Pharmacophore modeling and molecular docking studies on Pinus roxburghii as a target for diabetes mellitus. Advances in bioinformatics. 2014;2014.

47. Konappa N, Udayashankar AC, Krishnamurthy S, Pradeep CK, Chowdappa S, Jogaiah S. GC-MS analysis of phytoconstituents from Amomum nilgiricum and molecular docking interactions of bioactive serverogenin acetate with target proteins. Scientific Reports. 2020 Oct 2;10(1):1-23.

48. Nazir N, Zahoor M, Nisar M, Khan I, Karim N, Abdel-Halim H, Ali A. Phytochemical analysis and antidiabetic potential of Elaeagnus umbellata (Thunb.) in streptozotocin-induced diabetic rats: pharmacological and computational approach. BMC complementary and alternative medicine. 2018 Dec;18(1):1-6.

49. Da Hye Kim HA, Sohn HS, Kim JW, Choi JS. Potential of Icariin Metabolites from Epimedium koreanum Nakai as Antidiabetic Therapeutic Agents. Molecules: A Journal of Synthetic Chemistry and Natural Product Chemistry. 2017 Jun;22(6).

50. Zhu C, Niu H, Nie A, Bian M. Bioactivity-guided separation of potential $\alpha$-glycosidase inhibitor from clerodendranthusspicatus based on HSCCC coupled with molecular docking. Scientific Reports. 2021 Mar 25;11(1):1-2.

51. Choudhary DK, Mishra A. In vitro and in silico interaction of porcine $\alpha$-amylase with Vicia faba crude seed extract and evaluation of antidiabetic activity. Bioengineered. 2017 Jul 4;8(4):393-403

52. Herowati R, Widodo GP. Molecular Docking studies of chemical constituents of Tinospora cordifolia on glycogen phosphorylase. Procedia Chemistry. 2014 Jan 1;13:63-8.

53. Wang Y, Lin HQ, Xiao CY, Law WK, Hu JS, Ip TM, Wan DC. Using molecular docking screening for identifying hyperoside as an inhibitor of fatty acid binding protein 4 from a natural product database. Journal of functional foods. 2016 Jan 1;20:159-70.

54. Bharti SK, Krishnan S, Kumar A, Rajak KK, Murari K, Bharti BK, Gupta AK. Antihyperglycemic activity with DPP-IV inhibition of alkaloids from seed extract of Castanospermum australe: Investigation by experimental validation and molecular docking. Phytomedicine. 2012 Dec 15;20(1):24-31.

55. Odeyemi S, Dewar J. In vitro antidiabetic activity affecting glucose uptake in hepg2 cells following their exposure to extracts of Lauridiatetragona (Lf) RH Archer. Processes. 2020 Jan;8(1):33.

56. Hyun TK, Eom SH, Kim JS. Molecular docking studies for discovery of plant-derived a-glucosidase inhibitors. Plant Omics. 2014 May;7(3):166-70.

57. Melappa G, Channabasava R, Chandrappa CP, Sadananda TS. In vitro antidiabetic activity of three fractions of methanol extracts of Loranthus micranthus, identification of phytoconstituents by GC-MS and possible mechanism identified by GEMDOCK method. Asian Journal of Biomedical and Pharmaceutical Sciences. 2014 Jul 1;4(34):34.

58. Khanal P, Patil BM, Mandar BK, Dey YN, Duyu T. Network pharmacology-based assessment to elucidate the molecular mechanism of anti-diabetic action of Tinospora cordifolia. Clinical Phytoscience. 2019 Dec;5(1):1-9.

59. Angadi KK, Gundampati RK, Jagannadham MV, Kandru A. Molecular docking studies of guggultetrol from Nymphaea pubescens with target glucokinase (GK) related to type-II Diabetes. Journal of Applied Pharmaceutical Science. 2013 Feb 1;3(2):127.

60. Shah MR, Ishtiaq, Hizbullah SM, Habtemariam S, Zarrelli A, Muhammad A, Collina S, Khan I. Protein tyrosine phosphatase 1B inhibitors isolated from Artemisia roxburghiana. Journal of enzyme inhibition and medicinal chemistry. $2016 \mathrm{Jul}$ 3;31(4):563-7.

61. Zabidi NA, Ishak NA, Hamid M, Ashari SE, Mohammad Latif MA. Inhibitory evaluation of Curculigo latifolia on $\alpha$-glucosidase, DPP (IV) and in vitro studies in antidiabetic with molecular docking relevance to type 2 diabetes mellitus. Journal of Enzyme Inhibition and Medicinal Chemistry. 2021 Jan 1;36(1):109-21.

62. Phoopha S, Wattanapiromsakul C, Pitakbut T, DejAdisai S. Chemical constituents of Litsea elliptica and their alpha-glucosidase inhibition with molecular docking. Pharmacognosy Magazine. 2020 Apr 1;16(70):327. 